\title{
A globally convergent numerical method for a Coefficient Inverse Problem with backscattering data
}

\author{
Andrey V. Kuzhuget, Natee Pantong and Michael V. Klibanov \\ Department of Mathematics and Statistics \\ University of North Carolina at Charlotte Charlotte, NC 28223, USA \\ E-mails: \{akuzhuge, npantong, mklibanv\}@uncc.edu,
}

September 30, 2010

\begin{abstract}
A survey of recent results of the authors is presented. This survey is short due to space limitations. A Coefficient Inverse Problem for a hyperbolic PDE with backscattering data is considered. A globally convergent numerical method for this problem is presented. Analytical results are supported by computational ones.
\end{abstract}

\section{Introduction}

We present here a short survey of our recently obtained results of [17]. So, although some analytical results are formulated here, ideas of their proofs are only briefly outlined. In section 5 two numerical results are presented. Coefficient Inverse Problems (CIPs) for PDEs are both nonlinear and ill-posed. These two factors cause substantial difficulties for their numerical treatments. Still, because of a number of quite interesting applications, the devlopment numerical methods for CIPs is an important topic of the field of Inverse Problems. In the case of such applications as search for plastic antipersonnel land mines and detection of explosives the backscattering time resolved data is reasonable to use. Furthermore, these data should be collected either for a single location of the point source for a single direction of the incident plane wave. Indeed, this case presents both the most economical and the most practical scheme of data collection. Hence, exactly this case is considered in the current publication.

Because of the nonlinearity and ill-posedness combined, least squares functionals for CIPs, including Tikhonov regularization functionals, usually suffer from multiple local minima and ravines. The is the major stumbling block on the road of the development of reliable numerical methods. The classical regularization theory does not provide an answer on how to overcome this obstacle. Therefore, the theory of globally convergent numerical methods 
for CIPs needs to be developed. A numerical method for a CIP is called globally convergent if: (1) A rigorous theorem is proven which claims that this method provides a good approximation for the correct solution regardless on the availability of a good first guess for this solution, and (2) this theorem is confirmed in numerical experiments.

The development of globally convergent numerical methods, which are based on the layer stripping procedure with respect to the positive parameter $s>0$ of the Laplace transform of a hyperbolic PDE, has started in [1] and was continued in $[2,3,4,5,11,16,17]$. We call $s$ pseudo frequency. However, until the recent works $[16,17]$ only the case of complete data collection was considered. Namely, it was assumed that the data are given at the entire boundary of the domain of interest. As a result, Dirichlet boundary value problems were solved via the FEM for certain elliptic PDEs arising in this method. The case of backscattering data was considered only recently in the 1-D case in [16] and in the 2-D case in [17]. In the case of backscatteting data both Dirichlet and Neumann data are given only at the backscattering side of the boundary. Although it is reasonably to assume that the radiationlike boundary condition is given at the rest of the boundary, this condition is independent on the unknown coefficient and is therefore non-informative. So, the resulting over-determined boundary value problem is solved via the Quasi-Reversibility Method (QRM). The QRM was first proposed in [19] and was developed further in $[6,7,9,13,14,15]$. It has proven to be effective for solving boundary value problems with over-determined boundary conditions. The first application of the QRM to a CIP was published in [16] in the 1-D case and it was continued in [17] in the 2-D case.

Since CIPs are extremely challenging to handle, it is inevitable that an approximation is made in the globally convergent method of $[1,2,3,4,5,11,16,17]$. Namely, the high value of the pseudo frequency $\bar{s}$ is truncated. This truncation is similar with the truncation of high frequencies, which is routinely done in engineering. Because of this truncation, a new mathematical model was proposed in $[2,11]$ and it was actually numerically implemented, see subsection 7.1 of [11]. It was shown in these references that this model is neither better nor worse than the truncation of asymptotic series, which is a part of the classical Real Analysis.

We point out here to the publication [11], which has special importance for the method of $[1,2]$. Indeed, in the paper [11] the globally convergent method of [1] was independently verified on experimental data. Furthermore, even though these were blind experimental data, a surprisingly excellent accuracy of reconstructions of refractive indices of dielectric inclusions was consistently observed, see Tables 5 and 6 in [11]. It was shown in the follow up publication [5] that not only refractive indices but shapes of those inclusions can also be reconstructed with an excellent accuracy. Therefore, publications $[5,11]$ have provided an independent verification on real rather than synthetic data of both the globally convergent method of [1] and the subsequently developed two-stage numerical procedure of $[2,3,4]$.

As to the two-stage numerical procedure, the main reason why it was subsequently developed was that the above truncation of $\bar{s}$. Indeed, one cannot take $\bar{s}$ exceedingly large in real computations. Hence, although the globally convergent technique of $[1,2]$ provides a guaranteed good first approximation for the correct solution of a CIP, there is a room left for 
a refinement. So, the first stage is the globally convergent method of $[1,2]$. And the second one is the refinement stage. On the refinement stage the solution obtained on the first is taken as the starting point for a locally convergent technique. Indeed, it is well known that a good first approximation for the solution is the true key for any locally convergent numerical method, and the first stage provides that approximation. Furthermore, it follows from results of [4] that the resulting two-stage procedure converges globally. So, when working with the backscattering data, we use the gradient method on the second stage. It is explained in the recent work [18] why a locally convergent numerical method, which finds a minimizer of the Tikhonov functional, refines the first guess for the exact solution, as long as this guess is sufficiently close to that solution.

\section{Statements of Forward and Inverse Problems}

As the forward problem, we consider the Cauchy problem for a hyperbolic PDE

$$
\begin{aligned}
c(x) u_{t t} & =\Delta u \text { in } \mathbb{R}^{3} \times(0, \infty), \\
u(x, 0) & =0, u_{t}(x, 0)=\delta\left(x-x_{0}\right) .
\end{aligned}
$$

Equation (2.1) governs propagation of acoustic and electromagnetic waves. In the acoustical case $1 / \sqrt{c(x)}$ is the sound speed. In the 2 -D case of EM waves propagation, the dimensionless coefficient $c(x)=\varepsilon_{r}(x)$, where $\varepsilon_{r}(x)$ is the relative dielectric function of the medium, see [8], where this equation was derived from Maxwell's equations in the 2-D case. It should be also pointed out that although equation (2.1) cannot be derived from the Maxwell's system in the 3 -d case for $\varepsilon_{r}(x) \neq$ const., still it was successfully applied to the imaging from experimental data, and a very good accuracy of reconstruction was consistently observed [5, 11].

Since we work in this paper only with the backscattering data, we define the domain of our interest $\Omega$ as a rectangular prism as follows. Let $A, B=$ const. $>0$. Then

$$
\begin{aligned}
\Omega & =\left\{x_{1}, x_{2} \in(-B, B), z \in(0, A)\right\}, \partial \Omega=\cup_{i=1}^{3} \Gamma_{i}, \\
\Gamma_{1} & =\left\{x_{1}, x_{2} \in(-B, B), z=0\right\}, \Gamma_{2}=\left\{x_{1}, x_{2} \in(-B, B), z=A\right\}, \\
\Gamma_{3} & =\partial \Omega \backslash\left(\Gamma_{1} \cup \Gamma_{2}\right) .
\end{aligned}
$$

Here and below we denote for convenience $z:=x_{3}$. We also assume that in (2.2)

$$
x_{0} \in\{z<0\} \text {. Hence, } x_{0} \notin \bar{\Omega} \text {. }
$$

We assume that the coefficient $c(x)$ of equation (2.1) is such that

$$
\begin{aligned}
& c(x) \in[1, d], d=\text { const. }>1, c(x)=1 \text { for } x \in \mathbb{R}^{3} \backslash \Omega, \\
& c(x) \in C^{4}\left(\mathbb{R}^{3}\right) .
\end{aligned}
$$

We consider the following 
Inverse Problem. Suppose that the coefficient $c(x)$ satisfies (2.4) and (2.5), where the number $d>1$ is given. Assume that the function $c(x)$ is unknown in the domain $\Omega$. Determine the function $c(x)$ for $x \in \Omega$, assuming that the following two functions $g_{0}(x, t)$ and $g_{1}(x, t)$ are given for a single source position $x_{0}$ satisfying (2.4)

$$
u(x, t)=g_{0}(x, t), \partial_{\nu} u(x, t)=g_{1}(x, t), \forall(x, t) \in \Gamma_{1} \times(0, \infty),
$$

where $\nu$ is the unit outward normal vector at $\Gamma_{1}$.

As it is always the case in the theory of CIPs, these two functions model measurements of both he wave field and its normal derivative at the part $\Gamma_{1}$ of the boundary $\partial \Omega$. By (2.4) $\Gamma_{1}$ is the backscattering part of this boundary. In applications usually only the function $g_{0}(x, t)$ is measured. However, to get the function $g_{1}(x, t)$, we can approximately assume that $\Gamma_{1}=\{z=0\}$. Next, we can consider equation (2.1) with initial conditions (2.2) and the boundary condition $g_{0}$ as the initial boundary value problem in the half space $\{z<0\}$. Since by (2.6) the coefficient $c(x)=1$ in this half space, then we can uniquely solve this problems. Hence, we can uniquely determine the function $g_{1}(x, t)$ in $(2.7)$. After this we again consider $\Gamma_{1}$ only as a part of the plane $\{z=0\}$ when solving the inverse problem.

A priori knowledge of upper and lower bounds of the coefficient $c(x)$ corresponds well with the Tikhonov concept about the availability of a priori information for an ill-posed problem $[10,21]$. In applications the assumption $c(x)=1$ for $x \in \mathbb{R}^{3} \backslash \Omega$ means that the target coefficient $c(x)$ has a known constant value outside of the medium of interest $\Omega$. Another argument here is that one should bound the coefficient $c(x)$ from the below by a positive number to ensure that the operator in (2.1) is a hyperbolic one on all iterations of our numerical procedure. Since we do not impose a "smallness" condition on the number $d-1$, our numerical method is not a locally convergent one.

The question of uniqueness of this Inverse Problem is a well known long standing open problem. It is addressed positively only if the function $\delta\left(x-x_{0}\right)$ in $(2.2)$ is replaced with a function $f(x)$ such that $f(x) \neq 0, \forall x \in \bar{\Omega}$. Corresponding uniqueness theorems were proven via the method of Carleman estimates $[12,13]$. It is an opinion of the authors that because of many important applications, it makes sense to develop numerical methods, assuming that the question of uniqueness of the above inverse problem is addressed positively.

\section{The Globally Convergent Method}

\subsection{The Laplace transform}

Consider the Laplace transform of the functions $u$,

$$
w(x, s)=\int_{0}^{\infty} u(x, t) e^{-s t} d t, \text { for } s \geq \underline{s}=\text { const. }>0,
$$

where $\underline{s}$ is a certain number. It is sufficient to choose $\underline{s}$ such that the integral (3.1) would converge together with corresponding $(x, t)$-derivatives. We call the parameter $s$ pseudo 
frequency. Note that we do not use the inverse Laplace transform in our method, since approximations for the unknown coefficient are obtained in the pseudo frequency domain. We obtain

$$
\begin{aligned}
\Delta w-s^{2} c(x) w & =-\delta\left(x-x_{0}\right) \\
\lim _{|x| \rightarrow \infty} w(x, s) & =0 .
\end{aligned}
$$

As to the condition (3.3), it was proven in [3] that it is satisfied for sufficiently large values $\underline{s}$ as long as $s \geq \underline{s}$. Furthermore, it was also proven in [3] that for these values of $s$ we have

$$
w(x, s)>0 .
$$

It follows from the classic theory of elliptic PDEs that for every $s>\underline{s}$ there exists unique solution $w \in C^{5+\gamma}\left(\mathbb{R}^{3} \backslash\left\{\left|x-x_{0}\right|<\vartheta\right\}\right), \forall \vartheta>0$ of the problem (3.2), (3.3). Here $C^{k+\gamma}, \gamma \in$ $(0,1)$ are Hölder spaces, where $k \geq 0$ is an integer. The following Lemma helps to understand the asymptotic behavior of the function $w(x, s)$ at $s \rightarrow \infty$.

Lemma 3.1 [1]. Assume that conditions (2.5) and (2.6) are satisfied. Let the function $w(x, s) \in C^{5+\gamma}\left(\mathbb{R}^{3} \backslash\left\{\left|x-x_{0}\right|<\vartheta\right\}\right), \forall \vartheta>0$ be the solution of the problem (3.2), (3.3). Assume that geodesic lines, generated by the eikonal equation corresponding to the function $c(x)$ are regular, i.e. any two points in $\mathbb{R}^{3}$ can be connected by a single geodesic line. Let $l\left(x, x_{0}\right)$ be the length of the geodesic line connecting points $x$ and $x_{0}$. Then the following asymptotic behavior of the function $w$ and its derivatives takes place for $|\alpha| \leq 5, k=0,1, x \neq$ $x_{0}$

$$
D_{x}^{\alpha} D_{s}^{k} w(x, s)=D_{x}^{\alpha} D_{s}^{k}\left\{\frac{\exp \left[-s l\left(x, x_{0}\right)\right]}{f\left(x, x_{0}\right)}\left[1+O\left(\frac{1}{s}\right)\right]\right\}, s \rightarrow \infty,
$$

where $f\left(x, x_{0}\right)$ is a certain function and $f\left(x, x_{0}\right) \neq 0$ for $x \neq x_{0}$.

Remark 3.1. An separate question here is about an easily verifiable sufficient condition of the regularity of geodesic lines. In general, such a condition is unknown, except of the trivial case when the function $c(x)$ is close to a constant. Still, the theory of CIPs for equation (2.1) does not work without this assumption. We verify the asymptotic behavior of this lemma computationally, see subsection 7.2 of [1].

\subsection{The nonlinear integral differential equation}

It follows from (3.4) that one can consider the function $v=(\ln w) / s^{2}$. Substituting $w=$ $\exp \left(v s^{2}\right)$ in (3.2) and using (2.4) and (2.7), we obtain

$$
\begin{aligned}
\Delta v+s^{2}|\nabla v|^{2} & =c(x), \quad x \in \Omega, \\
\left.v\right|_{\Gamma_{1}} & =\varphi_{0}(x, s),\left.\quad v_{z}\right|_{\Gamma_{1}}=\varphi_{1}(x, s), \forall s \in[\underline{s}, \bar{s}],
\end{aligned}
$$

where functions $\varphi_{0}$ and $\varphi_{1}$ are obtained from Laplace transform (3.1) of functions $g_{0}$ and $g_{1}$ in an obvious manner. The term $\delta\left(x-x_{0}\right)$ is not present in (3.5) because $x_{0} \notin \bar{\Omega}$. An obvious inconvenience of equation (3.5) is that it contains two unknown functions $v$ and $c$. 
Thus, we now eliminate the function $c(x)$ from equation (3.5) via the differentiation with respect to $s$, using $\partial_{s} c(x)=0$. Introduce a new function

$$
q(x, s)=\frac{\partial v(x, s)}{\partial s}=\frac{\partial}{\partial s}\left(\frac{\ln w}{s^{2}}\right) .
$$

Assuming that the asymptotic behavior of Lemma 3.1 holds and using (3.7), we obtain

$$
\begin{aligned}
D_{x}^{\alpha}(v) & =O\left(\frac{1}{s}\right), D_{x}^{\alpha}(q)=O\left(\frac{1}{s^{2}}\right), s \rightarrow \infty ;|\alpha| \leq 2, \\
v(x, s) & =-\int_{s}^{\infty} q(x, \tau) d \tau .
\end{aligned}
$$

We represent the integral in (3.9) as

$$
v(x, s)=-\int_{s}^{\bar{s}} q(x, \tau) d \tau+V(x, \bar{s}),
$$

where $\bar{s}>\underline{s}$ is a large parameter which should be chosen in numerical experiments. Actually, $\bar{s}$ is one of regularization parameters of our method. In fact, we have truncated here the function $V(x, \bar{s})$, which we call the tail function,

$$
V(x, \bar{s})=-\int_{\bar{s}}^{\infty} q(x, \tau) d \tau .
$$

By (3.8)

$$
\left\|D_{\bar{s}}^{k} V(x, \bar{s})\right\|_{C^{2}(\bar{\Omega})}=O\left(\frac{1}{\bar{s}^{k+1}}\right), k=0,1 ; \bar{s} \rightarrow \infty .
$$

The tail function is unknown. Although by (3.11) this function is small for large values of $\bar{s}$, our numerical experience as well as the numerical experience of above cited previous publications on the globally convergent method shows one should that it would be better to somehow approximate the tail function updating it via an iterative procedure.

Thus, we obtain from (3.5), (3.7) and (3.10) the following nonlinear integral differential equation

$$
\begin{aligned}
& \Delta q-2 s^{2} \nabla q \cdot \int_{s}^{\bar{s}} \nabla q(x, \tau) d \tau+2 s\left[\int_{s}^{\bar{s}} \nabla q(x, \tau) d \tau\right]^{2} \\
& \quad+2 s^{2} \nabla q \nabla V-2 s \nabla V \cdot \int_{s}^{\bar{s}} \nabla q(x, \tau) d \tau+2 s(\nabla V)^{2}=0, x \in \Omega .
\end{aligned}
$$


Let $\psi_{0}(x, s)=\partial_{s} \varphi_{0}(x, s), \psi_{1}(s)=\partial_{s} \varphi_{1}(x, s)$. Then (3.6) implies that

$$
\left.q\right|_{\Gamma_{1}}=\psi_{0}(x, s),\left.q_{z}\right|_{\Gamma_{1}}=\psi_{1}(x, s), \forall s \in[\underline{s}, \bar{s}] .
$$

Using arguments of subsection 2.2 of [3] one can easily prove that for $s>\underline{s}$ and $\underline{s}$ is sufficiently large the function $w(x, s)$ tends to zero together with its appropriate $(x, s)$-derivatives as $|x| \rightarrow \infty$. Hence, assuming that numbers $A$ and $B$ in (2.3) are sufficiently large, we assume below that we have the following radiation condition

$$
\frac{\partial w}{\partial \nu}+\left.s w\right|_{\Gamma_{i}}=0, i=2,3 .
$$

Hence, using (3.7) we obtain for the function $q$

$$
\left.\frac{\partial q}{\partial \nu}\right|_{\Gamma_{i}}=\frac{1}{s^{2}}, i=2,3
$$

Since the Neumann boundary condition (3.14) is independent on the function $c(x)$, we use it only for the purpose of the stabilization of the QRM. The presence of integrals in (3.12) implies the nonlinearity, which is one of two main difficulties here. The second main difficulty is that the tail function $V$ is unknown. Hence, equation (3.12) contains two unknown functions: $q$ and $V$. The fact, which helps to prove the global convergence theorem, is that for sufficiently large values of $\bar{s}$ the tail function $V$ is small. The reason why we can approximate both functions $q$ and $V$ well is that we treat them differently: while we approximate the function $q$ via inner iterations, the function $V$ is approximated via outer iterations. If the functions $q$ and $V$ are approximated well from (3.12)-(3.14) together with their $x$-derivatives up to the second order, then the target unknown coefficient $c(x)$ is also approximated well from (3.5), where the function $v$ is computed from (3.10). Thus, below we focus on the following question: How to solve numerically the problem (3.12)-(3.14)?

\subsection{The sequence of over-determined boundary value problems}

To address the above question, we use a layer stripping procedure with respect to $s \in[\underline{s}, \bar{s}]$. We assume (approximately of course) that $q(x, s)$ as a piecewise constant function with respect to the pseudo frequency $s$. That is, we assume that there exists a partition $\underline{s}=$ $s_{N}<s_{N-1}<\ldots<s_{1}<s_{0}=\bar{s}$ of the interval $[\underline{s}, \bar{s}]$ with the sufficiently small grid step size $h=s_{i-1}-s_{i}$ such that $q(x, s)=q_{n}(x)$ for $s \in\left(s_{n}, s_{n-1}\right]$. We approximate the boundary conditions (3.13), (3.14) as

$$
\left.q_{n}\right|_{\Gamma_{1}}=\bar{\psi}_{0, n}(x),\left.\partial_{z} q_{n}\right|_{\Gamma_{1}}=\bar{\psi}_{1, n}(x),\left.\partial_{\nu} q_{n}\right|_{\Gamma_{i}}=\left(s_{n} s_{n-1}\right)^{-1}, i=2,3 .
$$

where $\bar{\psi}_{0, n}, \bar{\psi}_{1, n}$ and $\left(s_{n} s_{n-1}\right)^{-1}$ are averages of functions $\psi_{0}, \psi_{1}$ and $s^{-2}$ over the interval $\left(s_{n}, s_{n-1}\right)$. Rewrite (3.12) for $s \in\left(s_{n}, s_{n-1}\right]$ using this piecewise constant approximation. Then multiply the resulting approximate equation by the $s$-dependent Carleman Weight Function $(\mathrm{CWF})$ of the form

$$
\mathcal{C}_{n, \mu}(s)=\exp \left[-\mu\left|s-s_{n-1}\right|\right], s \in\left(s_{n}, s_{n-1}\right],
$$


and integrate with respect to $s \in\left(s_{n}, s_{n-1}\right]$. We obtain the following approximate equation in $\Omega$ for the function $q_{n}(x), n=1, \ldots, N$

$$
\begin{aligned}
L_{n}\left(q_{n}\right) & :=\Delta q_{n}-A_{1 n}\left(h \sum_{j=1}^{n-1} \nabla q_{j}-\nabla V_{n}\right) \nabla q_{n}= \\
& =B_{n}\left(\nabla q_{n}\right)^{2}-A_{2, n} h^{2}\left(\sum_{j=1}^{n-1} \nabla q_{j}\right)^{2}+2 A_{2, n} \nabla V_{n}\left(h \sum_{j=1}^{n-1} \nabla q_{j}\right)-A_{2, n}\left(\nabla V_{n}\right)^{2} .
\end{aligned}
$$

We have intentionally inserted dependence of the tail function $V_{n}$ from the iteration number $n$ here because we will approximate these functions iteratively. In (3.16) $A_{1, n}=A_{1, n}(\mu, h)$, $A_{2, n}=A_{2, n}(\mu, h), B_{n}=B_{n}(\mu, h)$ are certain numbers depending on $n, \mu$ and $h$, see specific formulas in [1]. It is convenient to set in (3.11)

$$
q_{0} \equiv 0 .
$$

It is clear that boundary value problems (3.15), (3.16) can be solved sequentially starting from $q_{1}$. Boundary boundary conditions (3.15) are over-determined. Hence, it is natural to apply a version of the QRM here, because the QRM finds "least squares" solutions in the case of over-determined boundary conditions.

Another important point here is that

$$
B_{n}(\mu, h)=O\left(\frac{1}{\mu}\right), \mu \rightarrow \infty .
$$

Hence, taking a sufficiently large value of $\mu$, we can ignore the nonlinear term $B_{n}\left(\nabla q_{n}\right)^{2}$ in $(3.16)$,

$$
B_{n}\left(\nabla q_{n}\right)^{2}:=0
$$

We have used $\mu=50$ in our computations and have used (3.18). Hence, (3.18) allows us to solve a linear problem for each $q_{n}$.

\subsection{The iterative process (in brief)}

Our algorithm reconstructs iterative approximations $c_{n, k}(x) \in C^{1}(\bar{\Omega})$ of the function $c(x)$. On the other hand, we need to solve the forward problem (3.2), (3.3) on each iterative step in order to obtain a new approximation for the tail. So, we need functions $c_{n, k}(x) \in C^{1}(\bar{\Omega})$ to ensure the $C^{2}$ - smoothness of the corresponding function $w\left(x, s ; c_{n, k}\right)$ in $\bar{\Omega}$. Here $w\left(x, s ; c_{n, k}\right)$ is the solution of the problem (3.2), (3.3) with $c:=c_{n, k}$. Also, we need to ensure that $c_{n, k}(x)=1$ outside of the domain $\Omega$. To ensure the latter, we extend each function $c_{n, k}(x)$ outside of $\Omega$, so that the resulting function $\widehat{c}_{n, k}(x)=1$ outside of $\Omega, \widehat{c}_{n, k}(x)=c_{n, k}(x)$ in a subdomain $\Omega^{\prime} \subset \subset \Omega$ and $\widehat{c}_{n, k} \in C^{1}\left(\mathbb{R}^{3}\right)$. In addition, to ensure the ellipticity of the operator in (3.2), we need to have $\widehat{c}_{n, k}(x) \geq$ const. $>0$ in $\mathbb{R}^{3}$. The latter is ensured by the global convergence Theorem 4.2. Indeed, since the exact solution $c^{*}$ of our inverse problem is such 
that $c^{*}(x) \geq 1$ (see (2.5)) and by Theorem 4.2 the function $c_{n, k}(x)$ is close to $c^{*}(x)$ in a subdomain $\Omega_{\varkappa} \subset \Omega$ defined in subsection 4.1 , then we show below that $\widehat{c}_{n, k}(x) \geq 1 / 2$ in $\mathbb{R}^{3}$.

So, we now describe a rather standard procedure of a proper extension outside of the domain $\Omega$. Choose a function $\chi(x) \in C^{\infty}\left(\mathbb{R}^{3}\right)$ such that

$$
\chi(x)=\left\{\begin{array}{c}
1 \text { in } \Omega^{\prime}, \\
\text { between } 0 \text { and } 1 \text { in } \Omega \backslash \Omega^{\prime}, \\
0 \text { outside of } \Omega .
\end{array}\right.
$$

The existence of such functions $\chi(x)$ is well known from the Real Analysis course. Define the target extension of the function $c_{n, k}$ as $\widehat{c}_{n, k}(x):=(1-\chi(x))+\chi(x) c_{n, k}(x), \forall x \in \mathbb{R}^{3}$. Hence, $\widehat{c}_{n, k}(x)=1$ outside of $\Omega$ and $\widehat{c}_{n, k} \in C^{1}\left(\mathbb{R}^{2}\right)$. Let a subdomain $\widetilde{\Omega} \subseteq \Omega$ and $\Omega^{\prime} \subset \subset \widetilde{\Omega}$. In Theorem $4.2 \widetilde{\Omega}:=\Omega_{\varkappa}$. Suppose that $c_{n, k}(x) \geq 1 / 2$ in $\widetilde{\Omega}$. Then $\widehat{c}_{n, k}(x) \geq 1 / 2$ in $\Omega$. Indeed, $\widehat{c}_{n, k}(x)-1 / 2=(1-\chi(x)) / 2+\chi(x)\left(c_{n, k}(x)-1 / 2\right) \geq 0$. In any case, we should solve the problem (3.2), (3.3) with $c:=\widehat{c}_{n, k}$.

On each iterative step $n$ we approximate both the function $q_{n}$ and the tail function $V_{n}$. Each iterative step requires an approximate solution of the boundary value problem (3.15), (3.16). This is done via the QRM, which is described in subsection 3.5. First, we choose an initial tail function $V_{1,1}(x) \in C^{2}(\bar{\Omega})$. As to the choice of $V_{1,1}$, it was taken as $V_{1,1} \equiv 0$ in [1]. In later publications $[2,3,4,5,11,16,17]$ the initial tail $V_{1,1}$ was taken as the one, which corresponds to the case $c(x) \equiv 1$, where $c(x):=1$ is the value of the unknown coefficient outside of the domain of interest $\Omega$, see (2.5). An observation was that while both these choices give the same result, the second choice leads to a faster convergence and both choices satisfy conditions of the global convergence theorem.

For each $q_{n}$ we have inner iterations with respect to tails. Consider the $k^{\text {th }}$ iteration for $q_{n}$. We approximate the function $q_{n, k}$ in this case, where $n, k \geq 1$. Recall that by (3.17) $q_{0} \equiv 0$. Suppose that functions $q_{i} \in H^{5}(\Omega), i=1, \ldots, n-1$ and tails $V_{1}, \ldots, V_{n-1}, V_{n, k} \in C^{2}(\bar{\Omega})$ are constructed. To construct the function $q_{n . k}$, we use the QRM to find an approximate solution of the following boundary value problem in $\Omega$

$$
\begin{gathered}
\Delta q_{n, k}-A_{1 n}\left(h \sum_{j=1}^{n-1} \nabla q_{j}-\nabla V_{n, k}\right) \nabla q_{n, k}= \\
-A_{2, n} h^{2}\left(\sum_{j=1}^{n-1} \nabla q_{j}\right)^{2}+2 A_{2, n} \nabla V_{n, k} \cdot\left(h \sum_{j=1}^{n-1} \nabla q_{j}\right)-A_{2, n}\left(\nabla V_{n, k}\right)^{2}, \\
\left.q_{n, k}\right|_{\Gamma_{1}}=\bar{\psi}_{0, n}(x),\left.\partial_{z} q_{n, k}\right|_{\Gamma_{1}}=\bar{\psi}_{1, n}(x),\left.\partial_{\nu} q_{n, k}\right|_{\Gamma_{i}}=\left(s_{n} s_{n-1}\right)^{-1}, i=2,3 .
\end{gathered}
$$

Hence, we obtain the function $q_{n, k} \in H^{5}(\Omega)$. By the embedding theorem $q_{n, k} \in C^{3}(\bar{\Omega})$. To reconstruct an approximation $c_{n, k}(x)$ for the function $c(x)$, we first, use the discrete version of $(3.10)$,

$$
v_{n, k}\left(x, s_{n}\right)=-h q_{n, k}(x)-h \sum_{j=1}^{n-1} q_{j}(x)+V_{n, k}(x) .
$$


Next, using (3.5), we set for $n \geq 1$

$$
c_{n, k}(x)=\Delta v_{n, k}\left(x, s_{n}\right)+s_{n}^{2}\left|\nabla v_{n, k}\left(x, s_{n}\right)\right|^{2}, x \in \Omega
$$

Hence, the function $c_{n, k} \in C^{1}(\bar{\Omega})$. Assuming that the exact solution of our Inverse Problem $c^{*} \geq 1$ in $\mathbb{R}^{3}$ (see (2.5)), it follows from Theorem 4.2 that $c_{n, k}(x) \geq 1 / 2$ in $\Omega_{\varkappa} \subset \Omega$. Next, we construct the function $\widehat{c}_{n, k}(x)$ as in the beginning of this section. In doing so we choose such a subdomain $\Omega^{\prime}$ which is a little bit less than $\Omega_{\varkappa}$. Thus, $\Omega^{\prime} \subset \subset \Omega_{\varkappa} \subset \Omega$. Hence, by (3.21) and (3.22) the function $\widehat{c}_{n, k} \in C^{\gamma}\left(\mathbb{R}^{2}\right)$. Next, we solve the forward problem (3.2), (3.3) with $c(x):=\widehat{c}_{n, k}(x)$ for $s:=\bar{s}$ and obtain the function $w_{n . k}(x, \bar{s})$. Next, we set for the new tail

$$
V_{n, k+1}(x)=\frac{\ln w_{n . k}(x, \bar{s})}{\bar{s}^{2}} \in C^{2}(\bar{\Omega})
$$

We continue these iterations with respect to tails until convergence occurs. We cannot prove this convergence. However, we have always observed numerically that functions $q_{n, k}, c_{n, k}$ and $V_{n, k}$ have stabilized at $k:=m_{n}$ for a certain $m_{n}$. So, assuming that they are stabilized, we set

$$
c_{n}(x):=c_{n, m_{n}}(x), q_{n}(x):=q_{n, m_{n}}(x), V_{n}(x):=V_{n, m_{n}}(x):=V_{n+1,1}(x) \text { for } x \in \Omega
$$

and repeat the above for $n:=n+1$. We stop iterations with respect to $n$ at $n:=N$.

\subsection{The quasi-reversibility method}

Let $H_{n, k}(x)$ be the right hand side of equation (3.19) for $n \geq 1$. Denote

$$
a_{n, k}(x)=A_{1, n}\left(h \sum_{j=1}^{n-1} \nabla q_{j}-\nabla V_{n, k}\right)
$$

Then the boundary value problem (3.19), (3.20) can be rewritten as

$$
\begin{gathered}
\Delta q_{n, k}-a_{n, k} \cdot \nabla q_{n, k}=H_{n, k} \\
\left.q_{n, k}\right|_{\Gamma_{1}}=\bar{\psi}_{0, n}(x),\left.\partial_{z} q_{n, k}\right|_{\Gamma_{1}}=\bar{\psi}_{1, n}(x),\left.\partial_{\nu} q_{n, k}\right|_{\Gamma_{i}}=\left(s_{n} s_{n-1}\right)^{-1}, i=2,3 .
\end{gathered}
$$

Since we have two boundary conditions rather then one at $\Gamma_{1}$, then the problem (3.24), (3.25) is over-determined. Hence, we find the "least squares" solution of this problem via the QRM. Specifically, we minimize the following Tikhonov functional

$$
J_{n, k}^{\alpha}(u)=\left\|\Delta u-a_{n, k} \cdot \nabla u-H_{n, k}\right\|_{L_{2}(\Omega)}^{2}+\alpha\|u\|_{H^{5}(\Omega)}^{2},
$$

subject to boundary condition (3.25), where the small regularization parameter $\alpha \in(0,1)$. Let $u(x)$ be the minimizer of this functional. Then we set $q_{n, k}(x):=u(x)$. Local minima do not occur here because (3.26) is the sum of square norms of two expressions, both of which 
are linear with respect to $u$. The second term in the right hand side of (3.26) is the Tikhonov regularization term. We use the $H^{5}(\Omega)$-norm here in order to ensure that the minimizer $u:=q_{n, k} \in C^{3}(\bar{\Omega})$, which implies in turn that functions $\widehat{c}_{n, k} \in C^{1}\left(\mathbb{R}^{2}\right)$.

Remarks 3.2. 1. In our computations we relax the smoothness assumptions via considering the $H^{2}(\Omega)$-norm in the second term in the right hand side of (3.26). This is possible because in computations we actually work with finite dimensional spaces. Specifically, we work with finite differences and do not use "overly fine" mesh, which means that dimensions of our "computational spaces" are not exceedingly large. In this case all norms are equivalent not only theoretically but practically as well. To the contrary, if the mesh would be too fine, then the corresponding space would be "almost" infinite dimensional. Indeed, we have observed in our computations that taking too fine mesh leads to poor quality images.

2. One may pose a question on why we would not avoid the QRM via using just one of two boundary conditions at $\Gamma_{1}$ in $(3.25)$, since we have the Neumann boundary condition at $\partial \Omega \backslash \Gamma_{1}$. However, in this case we would be unable to prove the $C^{3}-$ smoothness of the function $q_{n, k}$, because the boundary $\partial \Omega$ is not smooth. In the case of the Dirichlet boundary condition only $\left.q_{n, k}\right|_{\Gamma_{1}}$ we would be unable to prove smoothness even assuming that $\partial \Omega \in C^{\infty}$, because of the Neumann boundary condition at the rest of the boundary. In addition, since

conditions $\left.\partial_{\nu} q_{n, k}\right|_{\Gamma_{i}}=\left(s_{n} s_{n-1}\right)^{-1}$ are independent on the unknown coefficient, it seems to be that two boundary conditions rather than one at $\Gamma_{1}$ should provide a better reconstruction.

3. Furthermore, even though our convergence theory in Lemmata 4.1-4.3 and Theorems 4.1, 4.2 assures that we can ignore the boundary condition $\left.\partial_{\nu} q_{n, k}\right|_{\Gamma_{3}}=\left(s_{n} s_{n-1}\right)^{-1}$ at $\Gamma_{3}$, we use it in numerical studies, which brings in a better stability of the problem.

\section{Global Convergence}

In this section we briefly present results of our global convergence analysis, referring to [17] for details.

\subsection{Some estimates}

For brevity we scale variables in such a way that in section 4

$$
\Omega=\left\{x_{1}, x_{2} \in\left(-\frac{1}{4}, \frac{1}{4}\right), z \in\left(0, \frac{1}{2}\right)\right\}
$$

In this section $C=C(\Omega)>0$ denotes different positive constants depending only on the domain $\Omega$. Let $\lambda, \nu>2$ be two parameters. Introduce another Carleman Weight Function $K(z)$,

$$
K(z):=K_{\lambda, \nu}(z)=\exp \left(\lambda \rho^{-\nu}\right), \text { where } \rho(z)=z+\frac{1}{4}, z>0 .
$$

Note that $\rho(z) \in(0,3 / 4)$ in $\Omega$ and $\left.\rho(z)\right|_{\Gamma_{2}}=3 / 4$. Let the number $\varkappa \in(1 / 3,1)$. Denote $\Omega_{\varkappa}=\{x \in \Omega: \rho(z)<3 \varkappa / 4\}$. Hence, if $\varkappa_{1}<\varkappa_{2}$, then $\Omega_{\varkappa_{1}} \subset \Omega_{\varkappa_{2}}$. Also, $\Omega_{1}=\Omega$ and 
$\Omega_{1 / 3}=\varnothing$. Lemma 4.1 established the Carleman estimate for the Laplace operator. Although such estimates are well known [13, 20], this lemma is still new, because we use a non-standard CWF and also because only one, rather than conventional two zero boundary condition (4.1) is given at $\Gamma_{3}$. These were not done before.

Lemma 4.1 [17]. Fix a number $\nu:=\nu_{0}(\Omega)>2$. Consider functions $u \in H^{3}(\Omega)$ such that

$$
\left.u\right|_{\Gamma_{1}}=\left.u_{z}\right|_{\Gamma_{1}}=\left.u_{x}\right|_{\Gamma_{3}}=0 .
$$

Then there exists a constant $C=C(\Omega)>0$ such that for any $\lambda>2$ the following Carleman estimate is valid for all these functions

$$
\begin{aligned}
\int_{\Omega}(\Delta u)^{2} K^{2} d x d z \geq & \frac{C}{\lambda} \int_{\Omega}\left(u_{x x}^{2}+u_{z z}^{2}+u_{x z}^{2}\right) K^{2} d x d z+C \lambda \int_{\Omega}\left[(\nabla u)^{2}+\lambda^{2} u^{2}\right] K^{2} d x d z \\
& -C \lambda^{3}\|u\|_{H^{3}(\Omega)}^{2} \exp \left[2 \lambda\left(\frac{4}{3}\right)^{\nu_{0}}\right] .
\end{aligned}
$$

We now establish both existence and uniqueness of the minimizer of the functional (3.26). Lemma 4.2 is proven using the Riesz theorem.

Lemma 4.2 [17]. Suppose that in (3.26) $H_{n, k} \in L_{2}(\Omega)$ and that there exists a function $\Phi \in H^{5}(\Omega)$ satisfying boundary conditions (4.1). Assume that in (3.23) components $a_{n, k}^{(j)}, j=$ $1,2,3$ of the vector function $a_{n, k}$ are such that $a_{n, k}^{(j)} \in C(\bar{\Omega})$ and $\left\|a_{n, k}^{(j)}\right\|_{C(\bar{\Omega})} \leq 1$. Then there exists unique minimizer $u_{\alpha} \in H^{5}(\Omega)$ of the functional (3.26). Furthermore,

$$
\left\|u_{\alpha}\right\|_{H^{5}(\Omega)} \leq \frac{C}{\sqrt{\alpha}}\left(\left\|H_{n, k}\right\|_{L_{2}(\Omega)}+\|\Phi\|_{H^{5}(\Omega)}\right) .
$$

In the course of the proof of Theorem 4.2 we need

Lemma 4.3 [17]. Consider an arbitrary function $g \in H^{5}(\Omega)$. Let the function $u \in$ $H^{5}(\Omega)$ satisfies boundary conditions (4.1) as well as the variational equality

$$
\left(G_{n, k} u, G_{n, k} v\right)+\alpha[u, v]=\left(H_{n, k}, G_{n, k} v\right)+\alpha[g, v],
$$

for all functions $v \in H^{5}(\Omega)$ satisfying (4.1). Then

$$
\|u\|_{H^{5}(\Omega)} \leq \frac{\left\|H_{n, k}\right\|_{L_{2}(\Omega)}}{\sqrt{\alpha}}+\|g\|_{H^{5}(\Omega)} .
$$

Proof. Set in (4.2) $v:=u$ and use the Cauchy-Schwarz inequality.

Theorem 4.1 [17]. Consider an arbitrary function $g \in H^{5}(\Omega)$. Let $u \in H^{5}(\Omega)$ be the function satisfying (4.1) and (4.2). Let $\left\|a_{n, k}^{(j)}\right\|_{C(\bar{\Omega})} \leq 1$, where $a_{n, k}^{(j)}, j=1,2,3$ are two components of the vector function $a_{n, k}$ in (3.23). Choose an arbitrary number $\bar{\varkappa}$ such that $\bar{\varkappa} \in(\varkappa, 1)$. Consider the numbers $b_{1}, b_{2}$,

$$
b_{1}=\frac{1}{2\left(1+\left(1-\bar{\varkappa}^{\nu_{0}}\right)(3 \bar{\varkappa})^{-\nu_{0}}\right)}<\frac{1}{2}, b_{2}=\frac{1}{2}-b_{1}>0,
$$


where $\nu_{0}$ is the parameter of Lemma 4.1. Then there exists a sufficiently small number $\alpha_{0}=\alpha_{0}\left(\nu_{0}, \varkappa, \bar{\varkappa}\right) \in(0,1)$ such that for all $\alpha \in\left(0, \alpha_{0}\right)$ the following estimate holds

$$
\|u\|_{H^{2}\left(\Omega_{\varkappa}\right)} \leq C \frac{\left\|H_{n, k}\right\|_{L_{2}(\Omega)}}{\alpha^{b_{1}}}+\alpha^{b_{2}}\|g\|_{H^{5}(\Omega)} .
$$

Proof. Setting (4.2) $v:=u$ and using the Cauchy-Schwarz inequality, we obtain

$$
\left\|G_{n, k} u\right\|_{L_{2}(\Omega)}^{2} \leq F^{2}:=\left\|H_{n, k}\right\|_{L_{2}(\Omega)}^{2}+\alpha\|g\|_{H^{5}(\Omega)}^{2} .
$$

Note that $K^{2}(0)=\max _{\bar{\Omega}} K^{2}(z)=\exp \left(2 \lambda \cdot 4^{\nu_{0}}\right)$. Hence, $K^{-2}(0)\left\|K \cdot G_{n, k} u\right\|_{L_{2}(\Omega)}^{2} \leq F^{2}$. Clearly $\left(G_{n, k} u\right)^{2} K^{2} \geq(\Delta u)^{2} K^{2} / 2-C(\nabla u)^{2} K^{2}$. Hence,

$$
\int_{\Omega}(\Delta u)^{2} K^{2} d x d z \leq C \int_{\Omega}(\nabla u)^{2} K^{2} d x d z+K^{2}(0) F^{2} .
$$

Using Lemma 4.1, we can estimate the left hand side of (4.4) from the below for sufficiently large $\lambda>1$. Then the with $(\nabla u)^{2}$ in $(4.4)$ will be absorbed. We obtain

$$
\begin{aligned}
& \lambda K^{2}(0) F^{2}+C \lambda^{4}\|u\|_{H^{3}(\Omega)}^{2} \exp \left[2 \lambda\left(\frac{4}{3}\right)^{\nu_{0}}\right] \\
\geq & C \int_{\Omega}\left(u_{x x}^{2}+u_{z z}^{2}+u_{x z}^{2}+|\nabla u|^{2}+u^{2}\right) K^{2} d x d z \\
\geq & C \int_{\Omega_{\varkappa}}\left(u_{x x}^{2}+u_{z z}^{2}+u_{x z}^{2}+|\nabla u|^{2}+u^{2}\right) K^{2} d x d z \\
\geq & C \exp \left[2 \lambda\left(\frac{4}{3 \varkappa}\right)^{\nu_{0}}\right]\|u\|_{H^{2}\left(\Omega_{\varkappa}\right)}^{2} .
\end{aligned}
$$

Comparing the first line with the last in this sequence of inequalities, dividing by the exponential term in the last line, taking $\lambda \geq \lambda_{0}(C, \varkappa, \bar{\varkappa})>1$ sufficiently large and noting that for such $\lambda$

$$
\lambda^{4} \exp \left[-2 \lambda\left(\frac{4}{3 \varkappa}\right)^{\nu_{0}}\right]<\exp \left[-2 \lambda\left(\frac{4}{3 \bar{\varkappa}}\right)^{\nu_{0}}\right]
$$

we obtain a stronger estimate,

$$
\|u\|_{H^{2}\left(\Omega_{\varkappa}\right)}^{2} \leq C K^{2}(0) F^{2}+C\|u\|_{H^{3}(\Omega)}^{2} \exp \left[-2 \lambda\left(\frac{4}{3 \bar{\varkappa}}\right)^{\nu_{0}}\left(1-\bar{\varkappa}^{\nu_{0}}\right)\right]
$$

Applying Lemma 5.3 to the second term in the right hand side of (5.15), we obtain

$$
\|u\|_{H^{2}\left(\Omega_{\varkappa}\right)}^{2} \leq C F^{2}\left\{\exp \left(2 \lambda \cdot 4^{\nu_{0}}\right)+\alpha^{-1} \exp \left[-2 \lambda\left(\frac{4}{3 \bar{\varkappa}}\right)^{\nu_{0}}\left(1-\bar{\varkappa}^{\nu_{0}}\right)\right]\right\} .
$$


Since $\alpha \in\left(0, \alpha_{0}\right)$ and $\alpha_{0}$ is sufficiently small, we can choose sufficiently large $\lambda=\lambda(\alpha)$ such that

$$
\exp \left(2 \lambda \cdot 4^{\nu_{0}}\right)=\alpha^{-1} \exp \left[-2 \lambda\left(\frac{4}{3 \bar{\varkappa}}\right)^{\nu_{0}}\left(1-\bar{\varkappa}^{\nu_{0}}\right)\right] .
$$

We obtain from (4.7) that $2 \lambda \cdot 4^{\nu_{0}}=\ln \alpha^{-2 b_{1}}$. Hence, using (4.3) and (4.5)-(4.7) we obtain the desired result.

\subsection{Global convergence theorem}

We follow the concept of Tikhonov for ill-posed problems [21]. By this concept, one should assume that there exists an "ideal" exact solution of an ill-posed problem with the "ideal" exact data. Next, one should prove that the regularized solution is close to the exact one.

\subsubsection{Exact solution}

First, we need to introduce the definition of the exact solution. We assume that there exists a coefficient $c^{*}(x)$ satisfying conditions $(2.5),(2.6)$, and this function is the unique exact solution of our Inverse Problem with the exact data $\varphi_{0}^{*}(x, s), \varphi_{1}^{*}(x, s)$ in $(3.6)$. Let $w^{*}(x, s)$ be the Laplace transform (3.1) of the solution $u^{*}(x, t)$ of the problem $(2.1),(2.2)$ with $c(x):=c^{*}(x)$. Let

$$
v^{*}(x, s)=s^{-2} \ln \left[w^{*}(x, s)\right], q^{*}(x, s)=\partial_{s} v^{*}(x, s), V^{*}(x)=v^{*}(x, \bar{s}) .
$$

Hence, $q^{*}(x, s) \in C^{5+\gamma}(\Omega) \times C^{1}[\underline{s}, \bar{s}]$. The function $q^{*}$ satisfies equation (3.12) where $V$ is replaced with $V^{*}$. Boundary conditions for $q^{*}$ are the same as ones in (3.13), where functions $\psi_{0}(x, s), \psi_{1}(x, s)$ are replaced with the exact boundary conditions $\psi_{0}^{*}(x, s), \psi_{1}^{*}(x, s)$ for $s \in[\underline{s}, \bar{s}]$,

$$
\left.q^{*}\right|_{\Gamma_{1}}=\psi_{0}^{*}(x, s),\left.q_{z}^{*}\right|_{\Gamma_{1}}=\psi_{1}^{*}(x, s),\left.\partial_{n} q^{*}\right|_{\Gamma_{i}}=s^{-2}, i=2,3 .
$$

We call the function $q^{*}(x, s)$ the exact solution of the problem (3.12), (3.13) with the exact boundary conditions (4.8). For $n \geq 1$ let $q_{n}^{*}, \bar{\psi}_{0, n}^{*}$ and $\bar{\psi}_{1, n}^{*}$ be averages of functions $q^{*}, \psi_{0}^{*}$ and $\psi_{1}^{*}$ over the interval $\left(s_{n}, s_{n-1}\right)$. Hence, it is natural to assume that

$$
\begin{gathered}
q_{0}^{*} \equiv 0, \max _{1 \leq n \leq N}\left\|q_{n}^{*}\right\|_{H^{5}(\Omega)} \leq C^{*}, C^{*}=\text { const. }>1, \\
\left\|\bar{\psi}_{0, n}^{*}-\bar{\psi}_{0, n}\right\|_{H^{2}\left(\Gamma_{1}\right)}+\left\|\bar{\psi}_{1, n}^{*}-\bar{\psi}_{1, n}\right\|_{H^{1}\left(\Gamma_{1}\right)} \leq C^{*}(\sigma+h), \\
\max _{s \in\left[s_{n}, s_{n-1}\right]}\left\|q_{n}^{*}-q^{*}\right\|_{H^{5}(\Omega)} \\
\leq C^{*} h
\end{gathered}
$$

Here the constant $C^{*}=C^{*}\left(\left\|q^{*}\right\|_{C^{5}(\bar{\Omega}) \times C^{1}[\underline{s}, \bar{s}]}\right)$ depends only on the $C^{5}(\bar{\Omega}) \times C^{1}[\underline{s}, \bar{s}]$ norm of the function $q^{*}(x, s)$ and $\sigma>0$ is a small parameter characterizing the level of the error in the data $\psi_{0}(x, s), \psi_{1}(x, s)$. We use the $H^{5}(\Omega)$ norm because of the quasi-reversibility, 
see (3.26). The step size $h=s_{n-1}-s_{n}$ can also be considered as a part of the error in the data. In addition, by (4.8)

$$
\left.q_{n}^{*}\right|_{\Gamma_{1}}=\bar{\psi}_{0, n}^{*}(x),\left.\partial_{z} q_{n}^{*}\right|_{\Gamma_{1}}=\bar{\psi}_{1, n}^{*}(x),\left.\partial_{\nu} q_{n}^{*}\right|_{\Gamma_{i}}=\left(s_{n} s_{n-1}\right)^{-1}, i=2,3 .
$$

The function $q_{n}^{*}$ satisfies the following analogue of equation (3.12)

$$
\begin{aligned}
& \Delta q_{n}^{*}-A_{1, n}\left(h \sum_{i=1}^{n-1} \nabla q_{i}^{*}(x)-\nabla V^{*}\right) \cdot \nabla q_{n}^{*}=-A_{2, n} h^{2}\left(\sum_{i=1}^{n-1} \nabla q_{i}^{*}(x)\right)^{2} \\
& +2 A_{2, n} \nabla V^{*} \cdot\left(h \sum_{i=1}^{n-1} \nabla q_{i}^{*}(x)\right)-A_{2, n}\left(\nabla V^{*}\right)^{2}+F_{n}(x, h, \mu)
\end{aligned}
$$

Here $F_{n}(x, h, \mu)$ is the error function characterizing the error in the approximate equation (4.13). Since by (3.18) we have dropped the nonlinear term $B_{n}\left(\nabla q_{n}\right)^{2}$, we incorporate this term in the function $F_{n}(x, h, \mu)$. So, it is reasonable to assume that

$$
\left\|F_{n}(x, h, \mu)\right\|_{L_{2}(\Omega)} \leq C^{*}\left(h+\mu^{-1}\right) ; \mu h \geq 1 .
$$

\subsubsection{Global convergence theorem}

Assume that

$$
\bar{s}>1, \mu h \geq 1 \text {. }
$$

Then [1] $\max _{1 \leq n \leq N}\left\{\left|A_{1, n}\right|+\left|A_{2, n}\right|\right\} \leq 8 \bar{s}^{2}$. We assume for brevity that

$$
\bar{\psi}_{0, n}^{*}=\bar{\psi}_{0, n}, \bar{\psi}_{1, n}^{*}=\bar{\psi}_{1, n}
$$

The proof of Theorem 4.2 for the more general case (4.10) can easily be extended along the same lines. Still, we keep the parameter $\sigma$ of (4.10) as a part of the error in the data and incorporate it in the function $F_{n}$. Thus, we obtain instead of (4.14)

$$
\left\|F_{n}(x, h, \mu)\right\|_{L_{2}(\Omega)} \leq C^{*}\left(h+\mu^{-1}+\sigma\right) ; \mu h \geq 1
$$

We also recall that by the embedding theorem $H^{5}(\Omega) \subset C^{3}(\bar{\Omega})$ and

$$
\|f\|_{C^{3}(\bar{\Omega})} \leq C\|f\|_{H^{5}(\Omega)}, \forall f \in H^{5}(\Omega)
$$

The following global convergence theorem was proven in []

Theorem 4.2 [17]. Let the exact solution $c^{*}(x)$ satisfy conditions (2.5), (2.6). Suppose that conditions (4.8)-(4.13) and (4.15)- (4.17) are satisfied. Assume that for each $n \in[1, N]$ there exists a function $\Phi_{n} \in H^{5}(\Omega)$ satisfying boundary conditions (3.25). For any function 
$c \in C^{\gamma}\left(\mathbb{R}^{2}\right)$ such that $c(x) \in[1 / 2, d], c(x)=1$ in $\mathbb{R}^{3} \backslash \Omega$ consider the solution $w_{c}(x, \bar{s}) \in$ $C^{2+\gamma}\left(\mathbb{R}^{2} \backslash\left\{\left|x-x_{0}\right|<\vartheta\right\}\right), \forall \vartheta>0$ of the problem (3.2), (3.3). Let

$$
V_{c}(x)=\frac{\ln w_{c}(x, \bar{s})}{\bar{s}^{2}} \in C^{2+\gamma}\left(\mathbb{R}^{2} \backslash\left\{\left|x-x_{0}\right|<\vartheta\right\}\right), \vartheta>0
$$

be the corresponding tail function and $V_{1,1}(x, \bar{s}) \in C^{2}(\bar{\Omega})$ be the initial tail function. Suppose that the cut-off pseudo frequency $\bar{s}$ is so large that the following estimates hold

$$
\left\|V^{*}\right\|_{C^{1}(\bar{\Omega})} \leq \frac{\xi}{2},\left\|V_{1,1}\right\|_{C^{1}(\bar{\Omega})} \leq \frac{\xi}{2},\left\|V_{c}\right\|_{C^{1}(\bar{\Omega})} \leq \frac{\xi}{2}
$$

for any such function $c(x)$. Here $\xi \in(0,1)$ is a sufficiently small number. Introduce the parameter $\beta:=\bar{s}-\underline{s}$, which is the total length of the $s$-interval covered in our iterative process. Let $\alpha_{0}$ be so small that it satisfies the corresponding condition of Theorem 4.1. Let $\alpha \in\left(0, \alpha_{0}\right)$ be the regularization parameter of the $Q R M$. Assume that numbers $h, \sigma, \xi, \beta$, are so small that

$$
\begin{aligned}
& h+\mu^{-1}+\sigma+\xi \leq \beta, \\
& \beta \leq \frac{\sqrt{\alpha}}{136 \bar{s}^{2}\left(C^{*}\right)^{2} C_{1}},
\end{aligned}
$$

where the constant $C_{1} \in\left(1, C^{*}\right)$ depends only on the domain $\Omega$. Let $b_{2}$ be the number introduced in Theorem 4.1. Then the following estimates hold for all $\alpha \in\left(0, \alpha_{0}\right)$ and all $n \in[1, N]$

$$
\begin{aligned}
\left\|q_{n}\right\|_{H^{5}(\Omega)} & \leq 3 C^{*} \\
\left\|q_{n}-q_{n}^{*}\right\|_{H^{2}\left(\Omega_{\varkappa}\right)} & \leq 2 C^{*} \alpha^{b_{2}} \\
\left\|c_{n}-c^{*}\right\|_{C^{1}\left(\bar{\Omega}_{\varkappa}\right)} & \leq 2 C^{*} \alpha^{b_{2}}, c_{n} \geq \frac{1}{2} \text { in } \Omega_{\varkappa} .
\end{aligned}
$$

The proof of this theorem consists in sequential estimates of norms

$\left\|q_{n}-q_{n}^{*}\right\|_{H^{2}\left(\Omega_{\varkappa}\right)},\left\|c_{n}-c^{*}\right\|_{C^{1}\left(\bar{\Omega}_{\varkappa}\right)}$ using Theorem 4.1 and Lemmata 4.1-4.3. To estimate the norm $\left\|q_{n}-q_{n}^{*}\right\|_{H^{2}\left(\Omega_{\varkappa}\right)}$, we subtract equation (4.13) from equation (3.19) and consider the equation for the difference $q_{n}-q_{n}^{*}$. Next, the estimate of the norm $\left\|c_{n}-c^{*}\right\|_{C^{1}\left(\bar{\Omega}_{\varkappa)}\right)}$ is obtained via subtracting from (3.21) its analog for the function $v^{*}$ and using the estimate for $\left\|q_{n}-q_{n}^{*}\right\|_{H^{2}\left(\Omega_{\varkappa}\right)}$ as well (4.18).

\section{Remarks 4.1.}

1. Because of the term $\bar{s}^{-2}$ in inequalities (4.20), there is a discrepancy between these inequalities and (3.11). This discrepancy was discussed in detail in subsection 3.3 of [11] and in subsection 6.3 of [2], also see Introduction above. A new mathematical model proposed in these references allows the parameter $\xi$ in (4.19) to become infinitely small independently on the truncation pseudo frequency $\bar{s}$, also see discussion in the Introduction section above. We point out that this mathematical model was verified on experimental data. Indeed, actually 
the derivatives $\partial_{\bar{s}} V_{n, k}$ instead of functions $V_{n, k}$ were used in the numerical implementation of [11], see subsections 7.1 and 7.2 of [11]. It follows from (3.11) that one should expect that $\left\|\partial_{\bar{s}} V_{n, k}\right\|_{C^{2}(\bar{\Omega})}<<\left\|V_{n, k}\right\|_{C^{2}(\bar{\Omega})}=O(1 / \bar{s}), \bar{s} \rightarrow \infty$. Finally, we believe that, as in any applied problem, the independent verification on blind experimental data in [11] represents a valuable justification of this new mathematical model.

2. In our definition "global convergence" means that, given the above new mathematical model, there is a rigorous guarantee that a good approximation for the exact solution can be obtained, regardless on the availability of a good first guess about this solution. Furthermore, such a global convergence analysis should be confirmed by numerical experiments. So, Theorem 4.2, complemented by our numerical results below, satisfies these requirements.

3. The assumption of the smallness of the parameter $\beta=\bar{s}-\underline{s}$ is a natural one because equations (3.19) are actually generated by equation (3.12), which contains the nonlinearity in Volterra-like integrals. It is well known from the standard Ordinary Differential Equations course that solutions of nonlinear integral Volterra-like equations might have singularities on large intervals.

\section{$5 \quad$ Numerical Studies}

In these studies we have tested a simplified mathematical model of imaging of plastic land mines. The first main simplification of our model is that we consider the 2-D instead of the 3$\mathrm{D}$ case. Second, we ignore the air/ground interface, assuming that the governing PDE is valid on the entire 2-D plane. The third simplification is that we consider a plane wave instead of the point source in (2.1). This is because we have discovered that our algorithm works better with the case of the incident plane wave rather than with the case of a point source. The point source was considered above only for the convenience of analytical derivations due to Lemma 2.1.

\subsection{Modeling imaging of plastic land mines}

Let the ground be $\left\{x=\left(x_{1}, z\right): z>0\right\} \subset \mathbb{R}^{2}$. Suppose that a polarized electric field is generated by a plane wave, which is initialized at the point $\left(0, z^{0}\right), z^{0}<0$ at the moment of time $t=0$. The following hyperbolic equation can be derived from the Maxwell equations [8]

$$
\begin{aligned}
\varepsilon_{r}(x) u_{t t} & =\Delta u,(x, t) \in \mathbb{R}^{2} \times(0, \infty), \\
u(x, 0) & =0, u_{t}(x, 0)=\delta\left(z-z^{0}\right),
\end{aligned}
$$

where the function $u(x, t)$ is a component of the electric field. Recall that $\varepsilon_{r}(x)$ is the spatially distributed dielectric constant, see the beginning of section 2. We assume that the function $\varepsilon_{r}(x)$ satisfies the same conditions $(2.3),(2.4)$ as the function $c(x)$. The Laplace transform (2.5) leads to the following analog of the problem (2.6), (2.7)

$$
\begin{aligned}
\Delta w-s^{2} \varepsilon_{r}(x) w & =-\delta\left(z-z^{0}\right), \forall s \geq \underline{s}, \\
\lim _{|\mathbf{x}| \rightarrow \infty}\left(w-w_{0}\right)(x, s) & =0, \forall s \geq \underline{s},
\end{aligned}
$$


where

$$
w_{0}(z, s)=\frac{\exp \left(-s\left|z-z_{0}\right|\right)}{2 s}
$$

is such solution of equation (5.3) for the case $\varepsilon_{r}(x) \equiv 1$ that $\lim _{|z| \rightarrow \infty} w_{0}(z, s)=0$. Actually the function $w_{0}(z, s)$ corresponds to the case of the plane wave propagating in the free space.

It is well known that the maximal depth of an antipersonnel land mine does not exceed about $10 \mathrm{~cm}=0.1 \mathrm{~m}$. So, we model these mines as small squares with the $0.1 \mathrm{~m}$ length of sides, and their centers should be at the maximal depth of $0.1 \mathrm{~m}$. We set $\Omega=$ $\{x=(x, z) \in(-0.3 m, 0.3 m) \times(0 m, 0.6 m)\}$. Consider dimensionless variables $x^{\prime}=x / 0.1, z^{0 \prime}=$ $z^{0} / 0.1$. For brevity we keep the same notations for both these variables and the new domain $\Omega$,

$$
\Omega=(-3,3) \times(0,6) .
$$

We now use the values of the dielectric constant given at http://www.clippercontrols.com. Hence, $\varepsilon_{r}=5$ in the dry sand and $\varepsilon_{r}=22$ in the trinitrotoluene (TNT). We also take $\varepsilon_{r}=2.5$ in a piece of wood submersed in the ground. Since the dry sand should be considered as a background outside of our domain of interest $\Omega$, we introduce parameters $\varepsilon_{r}^{\prime}=\varepsilon_{r} / 5, s^{\prime}=$ $s \cdot 0.1 \cdot \sqrt{5}$, and again do not change notations. Hence, we now can assume that the following values of this scaled dielectric constant

$$
\varepsilon_{r}(\operatorname{dry} \text { sand })=1, \varepsilon_{r}(\mathrm{TNT}) \in[4,6] .
$$

In addition, centers of small squares modeling our targets should be in the following rectangle

$$
\{x=(x, z) \in[-2.5,2.5] \times[0.5,1.0]\} .
$$

The side of each of our small square should be 1, i.e. $10 \mathrm{~cm}$. The interval $z \in[0.5,1.0]$ in (5.7) corresponds to depths of centers between $5 \mathrm{~cm}$ and $10 \mathrm{~cm}$ and the interval $[-2.5,2.5]$ means that any such square is fully inside of $\Omega$.

The data for the inverse problem were generated by solving numerically equation (5.3) in the rectangle $R=[-4,4] \times[-2,8]$ with $z_{0}=-1$. By (5.5) $\Omega \subset R$. To avoid the singularity in $\delta\left(z-z^{0}\right)$, we actually solve in $R$ the equation for the function

$$
\widetilde{w}=w-w_{0}
$$

with the zero Dirichlet boundary condition for this function, see (5.4). We solve the resulting Dirichlet boundary value problem via the FDM with the uniform mesh size $h_{f}=0.0675$.

It is quite often the case in numerical studies that one should slightly modify the numerical scheme given by the theory, and so we did the same. Indeed, it is well known in computations that numerical results are usually more optimistic than analytical ones. We have modified our above algorithm via considering the function $\widetilde{v}=s^{-2} \cdot \ln \left(w / w_{0}\right)$. In other words, we have divided our solution $w$ of the problem (5.3), (5.4) by the initializing plane wave $w_{0}$. This has resulted in an insignificant modification of equations (3.19). We have observed in our computations that the function $w / w_{0}$ at the measurement part $\Gamma_{1} \subset \partial \Omega$ is poorly 
sensitive to the presence of abnormalities, as long as $s>1.2$. Hence, one should expect that the modified tail function for the function $\widetilde{v}$ should be small for $s>1.2$, which is exactly what is required by the above theory. For this reason, we have chosen the truncation pseudo frequency $\bar{s}=1.2$ and the initial tail function $V_{1,1} \equiv 0$.

\subsection{Some details of the numerical implementation of the globally convergent method}

We have generated the data for $s \in[0.5,1.2]$ with the grid step size $h=0.1$ in the $s$ direction. Since the grid step size in the $s$-direction is $h=0.1$ for $s \in[0.5,1.2]$, then $\beta=0.7$ and $N=7$. Also, we took the number of iterations with respect to the tail $m_{1}=m_{2}=\ldots=m_{N}:=$ $m=10$, since we have numerically observed the stabilization of functions $q_{n, k}, \varepsilon_{r}^{(n, k)}, V_{n, k}$ at $k=10$. In our computations we have relaxed the smoothness assumption in the QRM via taking in (3.26) the $H^{2}$-norm instead of the $H^{5}$-norm, see the first Remark 3.2. We have taken the following analog of the $H^{2}$-norm

$$
\left[\int_{\Omega}\left(u^{2}+(\nabla u)^{2}+u_{x_{1} x_{1}}^{2}+u_{z z}^{2}\right) d x d z\right]^{1 / 2},
$$

i.e. we have not included the mixed second derivative $\partial_{z} \partial_{x_{1}} u$.

Based on the experience of some earlier works on the QRM $[9,14,15]$ for linear illposed Cauchy problems, we have implemented the QRM via the FDM. Indeed, the FDM has allowed in [14] to image sharp peaks. On the other hand, the FEM of [9] did not let to image such peaks. So, we have written both terms under signs of norms of (3.26) in the FDM form. Next, to minimize the functional (3.26), we have used the conjugate gradient method. It is important that the derivatives with respect to the values of the unknown function at grid points should be calculated explicitly. This was done using the Kronecker symbol, see details in [14]. As soon as discrete values $\widetilde{\varepsilon}_{r}^{(n, k)}$ were computed, we have averaged each such value at the point $\left(x_{i}, z_{j}\right)$ over nine $(9)$ neighboring grid points, including the point $\left(x_{i}, z_{j}\right)$ : to decrease the reconstruction error. The resulting discrete function was taken as $\varepsilon_{r}^{(n, k)}$.

We have used the $49 \times 49$ mesh in $\Omega$. However, an attempt to use a finer $98 \times 98$ mesh led to a poor quality results. Most likely this is because the dimension of our above mentioned finite dimensional space was becoming too large, thus making it "almost" infinitely dimensional, which would require to use in (3.26) the $H^{5}$-norm instead of the $H^{2}$-norm, see the first Remark 3.2. The regularization parameter in (3.26) was taken $\alpha=0.04$.

We have made several sweeps over the interval $s \in[0.5,1.2]$ as follows. Suppose that

on the first $s$-sweep we have computed the discrete function $\varepsilon_{r}^{(1)}(x):=\varepsilon_{r}^{(N, 10)}(x)$, which corresponds to the last $s$-subinterval $\left[s_{N}, s_{N-1}\right]=[0.5,0.6]$. Hence, we have also computed the corresponding tail function $V^{(1)}(x)$. Next, we return to the first $s$-interval $\left[s_{1}, \bar{s}\right]=$ $[1.1,1.2]$, set $V_{1,1}^{(2)}(x):=V^{(1)}(x)$ and repeat the above algorithm. We kept repeating these 
$s$-sweeps $p$ times until either the stabilization has was observed, i.e.

$$
\left\|\varepsilon_{r}^{(p)}-\varepsilon_{r}^{(p-1)}\right\| \leq 10^{-5}
$$

or an "explosion" of the gradient of the functional $J_{n, k}^{\alpha}$ on the sweep number $p$ took place. "Explosion" means that

$$
\left\|\nabla J_{n, k}^{\alpha}\left(q_{n, k}^{(p)}\right)\right\| \geq 10^{5},
$$

for any appropriate values of indices $n, k$. Here and below $\|\cdot\|$ is the discrete $L_{2}(\Omega)-$ norm.

After the stopping criterion is achieved, we apply a post processing truncation procedure. We look for values of $\varepsilon_{r}>1$. Hence, in this procedure we first truncate to unity all values of $\varepsilon_{r}^{(p)}(x)$ which are less than 1 . Next, we truncate to unity $85 \%$ of the $\max \varepsilon_{r}^{(p)}(x)$, i.e. we truncate the treshhold. If we have several local maxima of $\varepsilon_{r}^{(p)}(x)$, then we apply the truncation procedure as follows. Let $\left\{x_{i}\right\}_{i=1}^{r} \subset \Omega$ be points where those local maxima are achieved, and values of those maxima are respectively $\left\{a_{i}\right\}_{i=1}^{r}$. Consider certain circles $\left\{B\left(x_{i}\right)\right\}_{i=1}^{r} \subset \Omega$ with the centers at points $\left\{x_{i}\right\}_{i=1}^{r}$ and such that $\bar{B}\left(x_{i}\right) \cap \bar{B}\left(x_{j}\right)=\varnothing$ for $i \neq j$. In each circle $B\left(x_{i}\right)$ set

$$
\widetilde{\varepsilon}_{r}^{(p)}(x):=\left\{\begin{array}{c}
\varepsilon_{r}^{(p)}(x) \text { if } \varepsilon_{r}^{(p)}(x) \geq 0.85 a_{i} \\
1, \text { otherwise. }
\end{array}\right.
$$

Next, for all points $x \in \Omega \backslash \cup_{i=1}^{r} B\left(x_{i}\right)$, we set $\widetilde{\varepsilon}_{r}^{(p)}(x):=1$. As a result, we have obtained the truncated function $\widetilde{\varepsilon}_{r}^{(p)}(x)$. Finally we set $\varepsilon_{r}^{g l o b}(x):=\widetilde{\varepsilon}_{r}^{(p)}(x)$. We consider the function $\varepsilon_{r}^{g l o b}(x)$ as the result obtained on the first stage of our two-stage numerical procedure. Next, we go to Stage 2. Figure 1 represents the typical example of the image obtained on the first stage of our two-stage numerical procedure.

\subsection{The second stage of our two-stage numerical procedure: a modified gradient method}

Recall that this method is used on the second stage of our two-stage numerical procedure. A complete, although space consuming derivation of this method, including the rigorous derivation of Frechét derivatives, can be done using the framework developed in [3, 4]. To save space, we derive it only briefly here. We call our technique the "modified gradient method" because rather than following usual steps of the gradient method, we find zero of the derivative Frechét of the Tikhonov functional via solving an equation with a contractual mapping operator.

Consider a wider rectangle $\Omega^{\prime} \supset \Omega$, where $\Omega^{\prime}=(-4,4) \times(0,6)$. We assume that both Dirichlet $\varphi_{0}$ and Neumann $\varphi_{1}$ boundary conditions in (2.7) are given on a wider interval $\Gamma_{1}^{\prime}=\{z=0\} \cap \bar{\Omega}^{\prime}, \Gamma_{1} \subset \subset \Gamma_{1}^{\prime}$. Let the function $\widetilde{w}$ be the one defined in (5.8). Hence, the following two functions $\widetilde{g}_{0}(x, s), \widetilde{g}_{1}(x, s)$ are known

$$
\begin{aligned}
\left.\widetilde{w}(x, s)\right|_{\Gamma_{1}^{\prime}} & =\widetilde{g}_{0}(x, s), \\
\left.\widetilde{w}_{z}(x, s)\right|_{\Gamma_{1}^{\prime}} & =\widetilde{g}_{1}(x, s)+e^{-s\left|z_{0}\right|},
\end{aligned}
$$




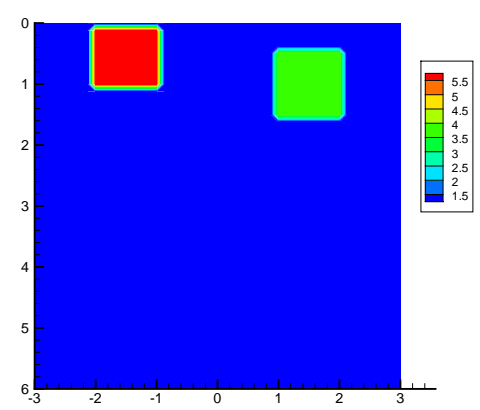

$1-\mathrm{a})$

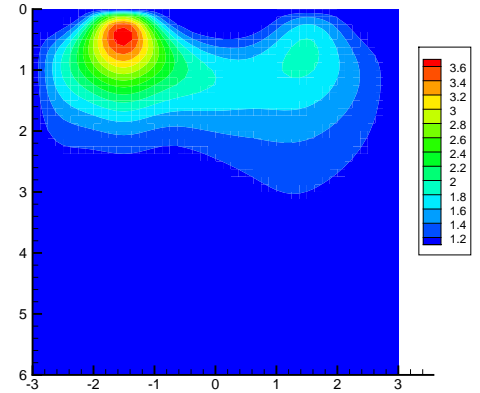

$1-b)$

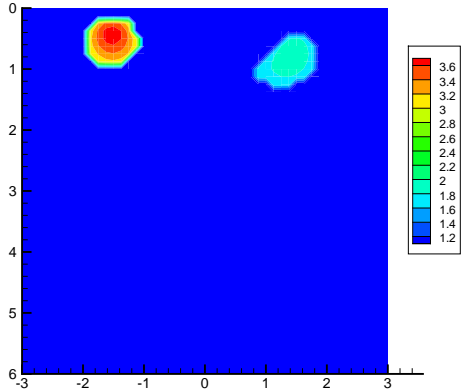

$1-c)$

Figure 1: A typical example of the image resulting from the globally convergent stage. The rectangle is the domain $\Omega$. This is the image of Test 1 (subsection 5.4). a) The correct coefficient. Inclusions are two squares with the same size $d=1$ of their sides, which corresponds to $10 \mathrm{~cm}$ in real dimensions. In the left square $\varepsilon_{r}=6$, in the right one $\varepsilon_{r}=4$ and $\varepsilon_{r}=1$ everywhere else, see (7.5) and (7.6). However, we do not assume knowledge of $\varepsilon_{r}(x)$ in $\Omega$. Centers of these squares are at $\left(x_{1}^{*}, z_{1}^{*}\right)=(-1.5,0.6)$ and $\left(x_{2}^{*}, z_{2}^{*}\right)=(1.5,1.0)$. b) The computed coefficient before truncation. Locations of targets are judged by two local maxima. So, locations are imaged accurately. However, the error of the computed values of the coefficient ?r in them is about 40\%. c) The image of b) after the truncation procedure, see the text.

Using (5.4), we obtain the following approximate boundary condition for the function $\widetilde{w}$

$$
\left.\partial_{\nu} \widetilde{w}\right|_{\partial \Omega^{\prime} \backslash \Gamma_{1}^{\prime}}=0 .
$$

In addition, by (5.3)

$$
\Delta \widetilde{w}-s^{2} \varepsilon_{r}(x) \widetilde{w}-s^{2}\left(\varepsilon_{r}(x)-1\right) w_{0}(z, s)=0, \text { in } \Omega^{\prime} .
$$

So, we now consider the solution of the boundary value problem (5.10)-(5.12). We want to find such a coefficient $\varepsilon_{r}(x)$, which would minimize the following Tikhonov functional

$$
\begin{aligned}
T\left(\varepsilon_{r}\right)= & \frac{1}{2} \int_{a}^{b} \int_{\Gamma_{1}^{\prime}}\left(\widetilde{w}(x, 0, s)-\widetilde{g}_{0}(x, s)\right)^{2} d x d s+\frac{\theta}{2}\left\|\varepsilon_{r}-\varepsilon_{r}^{g l o b}\right\|_{L_{2}(\Omega)}^{2} \\
& +\int_{a}^{b} \int_{\Omega^{\prime}} \lambda\left[\Delta \widetilde{w}-s^{2} \varepsilon_{r}(x) \widetilde{w}-s^{2}\left(\varepsilon_{r}(x)-1\right) w_{0}(z, s)\right] d x d s,
\end{aligned}
$$

where $\theta>0$ is the regularization parameter and $\lambda(x, s)$ is the solution of the so-called "adjoint problem",

$$
\Delta \lambda-s^{2} \varepsilon_{r}(x) \lambda=0, \text { in } \Omega^{\prime}
$$




$$
\left.\lambda_{z}\right|_{\Gamma_{1}^{\prime}}=\widetilde{w}(x, 0, s)-\widetilde{g}_{0}(x, s),\left.\partial_{\nu} \lambda\right|_{\partial \Omega^{\prime} \backslash \Gamma_{1}^{\prime}}=0 .
$$

Because of (5.12), the second line in (5.13) equals zero. Although boundary value problems (5.10)-(5.12) and (5.14), (5.15) are considered in the domain $\Omega^{\prime}$ with a non-smooth boundary, we have always observed in our computations existence of numerical solutions with no singularities. Although, by the Tikhonov theory, one should consider a stronger $H^{k}$-norm of $\varepsilon_{r}-\varepsilon_{r}^{g l o b}$ in (5.13) [21], we have found in our computations that the simpler $L_{2}-$ norm is sufficient. This is likely because we have worked computationally worked with not too many grid points. The same was observed in $[2,3,4,5]$. We obtain the following expression for the Fréchet derivative $T^{\prime}\left(\varepsilon_{r}\right)$ of the functional $T$

$$
T^{\prime}\left(\varepsilon_{r}\right)(x)=\theta\left(\varepsilon_{r}-\varepsilon_{r}^{g l o b}\right)(x)-\int_{a}^{b} s^{2}\left[\lambda\left(\widetilde{w}+w_{0}\right)\right](x, s) d s, x \in \Omega .
$$

Hence, to find a minimizer, one should solve the equation $T^{\prime}\left(\varepsilon_{r}\right)=0$. We solve it iteratively as follows

$$
\varepsilon_{r}^{n}(x)=\varepsilon_{r}^{g l o b}(x)+\frac{1}{\theta} \int_{a}^{b} s^{2}\left[\lambda\left(\widetilde{w}+w_{0}\right)\right]\left(x, s, \varepsilon_{r}^{n-1}\right) d s, x \in \Omega,
$$

where functions $\widetilde{w}\left(x, s, \varepsilon_{r}^{n-1}\right)$ and $\lambda\left(x, s, \varepsilon_{r}^{n-1}\right)$ are solutions of problems (5.10)-(5.12) and (5.14), (5.15) respectively with $\varepsilon_{r}(x):=\varepsilon_{r}^{n-1}(x)$. One can easily prove that one can choose the number $(b-a) / \theta$ so small that the operator in (5.16) will get the contractual mapping property. In our computations we took $a=0.01, b=0.05, \theta=0.15$. We have iterated in (5.16) until the stabilization has occurred, i.e. we have stopped iterations as soon as $\left\|\varepsilon_{r}^{n}-\varepsilon_{r}^{n-1}\right\| /\left\|\varepsilon_{r}^{n-1}\right\| \leq 10^{-5}$, where $\|\cdot\|$ is the discrete $L_{2}(\Omega)$ norm. Then we set that our computed solution is $\varepsilon_{r}^{n}(x)$.

\subsection{Two numerical results}

We present results of two numerical tests. Test 1 is in 2-D [17] and Test 2 is in 3-D. One can find one more test in 2-D in [17]. In our numerical tests we have introduced the multiplicative random noise in the boundary data using the following expression

$$
w_{\sigma}\left(x_{i}, 0, s_{j}\right)=w\left(x_{i}, 0, s_{j}\right)[1+\omega \sigma], i=0, \ldots, M ;, j=1, . ., N,
$$

where $w\left(x_{i}, 0, s_{j}\right)$ is the value of the computationally simulated function $w$ at the grid point $\left(x_{i}, 0\right) \in \Gamma_{1}^{\prime}$ and at the value $s:=s_{j}$ of the pseudo frequency, $\omega$ is a random number in the interval $[-1,1]$ with the uniform distribution, and $\sigma=0.05$ is the noise level. Hence, the random error is presented only in the Dirichlet data (5.9) but not in the Neumann data (5.10).

Test 1 (the 2-D case) [17]. We test our numerical method for the case of two squares with the same length $l=1$ of their sides. In the left square $\varepsilon_{r}=6$, in the right one $\varepsilon_{r}=4$ and $\varepsilon_{r}=1$ everywhere else, see (5.7). Centers of these squares are at $\left(x_{1}^{*}, z_{1}^{*}\right)=(-1.5,0.6)$ 
and $\left(x_{2}^{*}, z_{2}^{*}\right)=(1.5,1.0)$. This models two plastic land mines whose centers are at $6 \mathrm{~cm}$ and $10 \mathrm{~cm}$ depths and the size of each mine is $10 \mathrm{~cm} \times 10 \mathrm{~cm}$. However, we do not assume a priori in our algorithm neither the presence of these squares nor a knowledge of $\varepsilon_{r}(x)$ at any point of the rectangle $\Omega$. Figure 1 displays the result obtained on the globally convergent stage and Figure 2-b) displays the result obtained by the gradient method, which has used the image of Figure 1-c) as the starting point of iterations. Locations of both inclusions are imaged well. The computed function $\varepsilon_{r}(x)=1$ outside of these inclusion. The maximal value of the computed coefficient $\varepsilon_{r}(x)$ in the left inclusion is 6 and in the right inclusion is 4 , which is exactly the same as they should be. Both these values are achieved at correct centers of these inclusions, $\left(x_{1}^{*}, z_{1}^{*}\right)=(-1.5,0.6)$ and $\left(x_{2}^{*}, z_{2}^{*}\right)=(1.5,1.0)$. Hence, Figure 2 -b) displays quite an accurate image of the unknown coefficient.

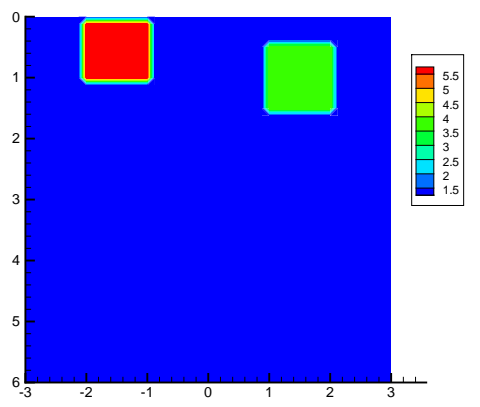

$2-\mathrm{a})$

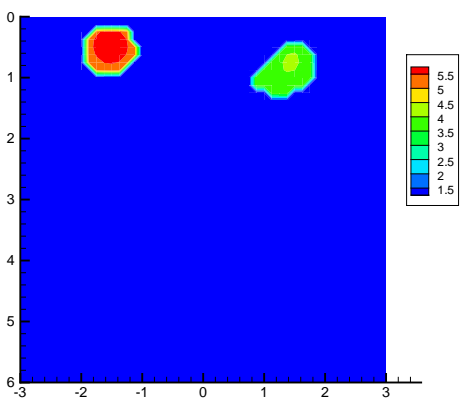

$1-\mathrm{b})$

Figure 2: Test 1. The image obtained on the globally convergent stage is displayed on Fig. 1-c). a) The correct image. Centers of small squares are at $\left(x_{1}^{*}, z_{1}^{*}\right)=(-1.5,0.6)$ and $\left(x_{2}^{*}, z_{2}^{*}\right)=(1.5,1.0)$ and values of the target coefficient are $\varepsilon_{r}=6$ in the left square, $\varepsilon_{r}=4$ in the right square and $\varepsilon_{r}=1$ everywhere else. b) The imaged coefficient $\varepsilon_{r}(x)$ resulting of our two-stage numerical procedure. Both locations of centers of targets and values of $\varepsilon_{r}(x)$ at those centers are imaged accurately.

Test 2 (the 3-D case). Our studies in 3-D are not complete yet at the time of the submission of this paper. So, we provide only a preliminary result here. An additional effort is required to finalize the $3-\mathrm{D}$ case. We have imaged a 3-D analog of the left inclusion of Figure 2-a). Namely, instead of the square whose sides are of the length 1 , we have imaged the rectangular prism $(x, y, z) \in(-2,2) \times(-3,3) \times(0,3)$. The center of the small cube to be imaged is at the point $(0,-1.5,0.6)$. The length of the side of this cube is 1 . Next, $\varepsilon_{r}=6$ inside of this cube and $\varepsilon_{r}=1$ outside of it. A certain post processing procedure was applied. Figure 3 displays the final result. The center of the inclusion is imaged with a good accuracy. The maximal value of the computed function $\varepsilon_{r}(x)$ is $\varepsilon_{r}=6$ and it is achieved at this center. Also, $\varepsilon_{r}=1$ outside of the imaged inclusion.

\section{Acknowledgment}




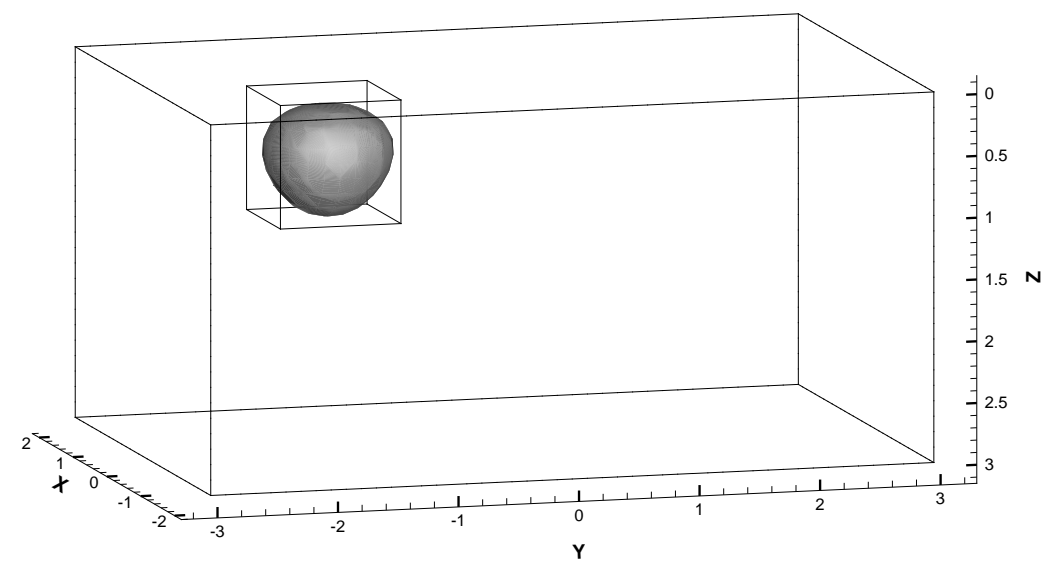

Figure 2: The final result for the 3-D case (Test 2). The center of the inclusion is imaged with a good accuracy. The maximal value of the computed function $\varepsilon_{r}(x)$ is $\varepsilon_{r}=6$ and it is achieved at this center. Also, $\varepsilon_{r}=1$ outside of the imaged inclusion.

This work was supported by the U.S. Army Research Laboratory and U.S. Army Research Office under grant number W911NF-08-1-0470.

\section{References}

[1] L. Beilina and M. V. Klibanov, A globally convergent numerical method for a coefficient inverse problem, SIAM J. Sci. Comp., 31, 478-509, 2008.

[2] L. Beilina and M. V. Klibanov, Synthesis of global convergence and adaptivity for a hyperbolic coefficient inverse problem in 3D, J. Inverse and Ill-Posed Problems, 18, 85-132, 2010.

[3] L. Beilina and M. V. Klibanov, A posteriori error estimates for the adaptivity technique for the Tikhonov functional and global convergence for a coefficient inverse problem, Inverse Problems, 26 (2010), 045012.

[4] L. Beilina, M. V. Klibanov and M.Yu. Kokurin, Adaptivity with relaxation for ill-posed problems and global convergence for a coefficient inverse problem, J. Mathematical Sciences, 167, 279-325, 2010.

[5] L. Beilina and M. V. Klibanov, Reconstruction of dielectrics from experimental data via a hybrid globally convergent/adaptive inverse algorithm, Inverse Problems, accepted for publication, 2010.

[6] L. Bourgeois, A mixed formulation of quasi-reversibility to solve the Cauchy problem for Laplace's equation, Inverse Problems, 21, 1087-1104, 2005. 
[7] L. Bourgeois, Convergence rates for the quasi-reversibility method to solve the Cauchy problem for Laplace's equation, Inverse Problems, 22, 413-430, 2006.

[8] M. Cheney and D. Isaacson, Inverse problems for a perturbed dissipative half-space, Inverse Problems, 11, 865-888, 1995.

[9] C. Clason and M.V. Klibanov, The quasi-reversibility method for thermoacoustic tomography in a heterogeneous medium, SIAM J. Sci. Comp., 30, 1-23, 2007.

[10] H.W. Engl, M. Hanke and A. Neubauer, Regularization of Inverse Problems, Kluwer Academic Publishers, Boston, 2000.

[11] M.V. Klibanov, M.A Fiddy, L. Beilina, N. Pantong and J. Schenk, Picosecond scale experimental verification of a globally convergent algorithm for a coefficient inverse problem, Inverse Problems, 26, 045003, 2010.

[12] M.V. Klibanov, Inverse problems and Carleman estimates, Inverse Problems, 8, 575596, 1991.

[13] M.V. Klibanov and A. Timonov, Carleman Estimates for Coefficient Inverse Problems and Numerical Applications, VPS, The Netherlands, 2004.

[14] M.V. Klibanov, A.V. Kuzhuget, S.I. Kabanikhin and D.V. Nechaev, A new version of the quasi-reversibility method for the thermoacoustic tomography and a coefficient inverse problem, Applicable Analysis, 87, 1227-1254, 2008.

[15] M.V. Klibanov and F. Santosa, A computational quasi-reversibility method for Cauchy problems for Laplace's equation, SIAM J. Appl. Math., 51, 1653-1675, 1991.

[16] A.V. Kuzhuget and M.V. Klibanov, Global convergence for a 1-D inverse problem with application to imaging of land mines, Applicable Analysis, 89, 1227-1254, 2008.

[17] A.V. Kuzhuget, L. Beilina, M.V. Klibanov and V.G. Romanov, Global convergence and quasi-reversibility for a coefficient inverse problem with backscattering data, available on-line at http://www.ma.utexas.edu/mp_arc, 2010.

[18] M.V. Klibanov, A.B. Bakushinskii and L. Beilina, Why a minimizer of the Tikhonov functional is closer to the exact solution than the first guess, available on-line at http://www.ma.utexas.edu/mp_arc, 2010.

[19] R. Lattes and J.-L. Lions, The Method of Quasireversibility: Applications to Partial Differential Equations, Elsevier, New York, 1969.

[20] M.M. Lavrent'ev, V.G. Romanov and S.P. Shishatskii, Ill-Posed Problems of Mathematical Physics and Analysis, AMS, Providence, RI, 1986.

[21] A.N. Tikhonov, A.V. Goncharsky, V.V. Stepanov and A.G. Yagola, Numerical Methods for Solutions of Ill-Posed Problems, Kluwer, London, 1995. 